Cas d'une distribution de force motrice. - Si notre entreprise obtient, comme clle le desire, le droil de poser des canalisations spéciales pour une distribution de force motrice (comme celle de Lijon et celle projetée pour Londres), on peut estimer comme il suit les dépenses supplementaires, en supposant 70000 kilowmoyens vendus aux abonnés.

Frais detablissement. -.. Lignes is 10000 et 2000 volts pour 50000 kilow. vendus, à 300 francs......

Transformateurs et lignes sccondaires it 500 volts pour 20000 kilow. it 500 francs............. 10.000.000 "

Total....... $25.000 .000 \mathrm{fr}$.

ce qui donne une moyenne de 350 francs par kilow. vendu.

l'rix de venle par lillowalt-an. - Les frais d'intérèt et d'amortissement et d'exploitation $=10 \%$ de 350 francs $=35$ francs.

Ces charges fixes seronl ainsi portées de 64 francs (chiffre donne plus haut) i 100 frantes en chiffe rond. Les charges mobiles scraient augmentées d'environ 1 centime.

On remarquera que, pour abaisser le prix rle la force motrice chez louvrier, on repartira les frais de distubution sur tous les consommaleurs.

En chiffre rond, le prix de vente chez l'abonné de force motrice devient ainsi, en francs, par kilowatt-an:

$$
P=100+0,015 n
$$

$n$ élant le nombre d'heures. Il on résulte pour le kilowatt-an $P$ et le kilowaltheure $p$ les prix suivants :

$\Lambda$ vec 3000 kilow. par an, $P=1$ íj fl. soit 105 fr. le cheval ; ef $p=0,018$ environ.

Avec (jolo kilow. par an, $P=190$ fr. soit $13 \pi$ fr. le cheval ; et $p=0,0.3$ environ.

Aures alantages grineraux de la nouvelle solution. - Les avantages de la nourclle solution sont considerables, au point de vue gênéral.

$1^{\circ}$ Lat durée el les frais d'installation se trouveront diminués par la suppression des longs tumnels prévus dans le premier projet : on evitera ainsi tout chomage partiel pouvant résulter de réparálions à l'un des tunnels.

$\therefore$ Lílulilisalion de la puissance du Rhone alleindra son maximurn ; on réalisera ainsi le but auguel dojt tendre touls administrution soncieuse des intercts de la collectivilie, it savoir tirer pour celle collectivite le maximum darantanes possibles des richesses naturelles : aucum des projels anterieurement conçus pour l'ulitisation des caux du Rhone ne presente ces memes avantages ; melui-ci est réellement un projet d'utilité publicqoe maxima.

Tous les excedents de force disponilyle penxlant la période des hantes eatu pourronl otre ainsi employes, soit it fournir une plus grande qumité donergie is bon mirché is Paris, ou dans les villes traversces par le projet, soit it almenter cerlaines installatinns locales, ou i crécr de nouvelles applicalions de lélactrochimic. Il est inléressant de remarquer que les haules caux du Thone correspontent aux tiages des rivieres des régions traversies et guon pourrait satisfaire aux besoins d'énergie de ces rérgions atu moment où elles en ont le plus besoin, sans gêner le transport sur Paris.

Les fruis d'exploitation de l'usine unique seront très minimes en comparaison de ceux des trois usines qu'exigerait la division du Rhone en retenues partielles. Le prix et le rendenent des turbines et dynamos est réduit d'une façon importante par l'emploi d'une haute chule unique au lieu de plusieurs. Les dépenses d'installations seront faites senlement au fur et it mesure que le placement de l'encrgie correspondante sera assurée, afin de réduire au minimum le capital improductif.

$3^{\circ}$ Id sera mis fin aux érosions du lit du Rhone entre la frontière suisse et Génissiat ; une voie d'eau naviusable sera créée entre ces deux points : pent-etre pourrait-elle meme plus tard etre ulilisce, si l'on créait ì Grémissiat un ascenseur à bateaux, et il deviendrait alors facile d'utablir la communication par eau entre Lyon et Genève, et de réaliser ainsi un reve conşu il y a plus d'un siècle par Céard, l'auteur célébre de la route du Simplon; on aplanira ainsi la plus grande difficulté de la mise en relation de la Suisse avec Iyon et la Méditerranée.

$4^{\circ}$ Enfin, l'utilisation du lac du Bourget, comme réservoir compensateur, permettrait non seulement de rendre insensibles les variations du régime du Rhone provoquées par les valliations diurnes du fonctionnement de l'usine, mais encore d'ameliorer la navigabilité du Rhône à Lyon.

\section{SYSTÈME DE SIGNES CONVENTIONNELS} pour la représentation des Appareils et Installations électriques (1)

Dans le courant de 1905, la sixième Section de votre Comité a été saisie, par notre collègue $M$. Vedoveli, d'une proposition tendant à mettre à l'étude un système de signes conventionnels pour la représentation des appareils et installations électriques.

M. Vedovelli soumettait, en même temps qu'une liste d'appareils très détaillée, un grand nombre de signes s'y rapportant, susceptibles d'un emploi courant et sur lesquels pouvait s'exercer la discussion et le choix de la Société des Electriciens. Ce tableau préparatoire constituait une base de travail pour un projet d'un caractère plus général, dont l'étude fut conflée à une sous-commission composée de MM. Aliamet, Boistel, Hospitalier et Pérot, et dont j'eus le plaisir de faire partie.

Les propositions de cette sous-commission furent ensuite discutées en détail sous la direction de notre sympathique et éminent Président de la sixic̀me Section, M. Guillaume, au cours des séances régulières de cette Section. La question, quoique d'un intérêt purement professionnel, mérite cependant, croyons-nous, un moment d'attention.

J'ai l'honneur de vous rapporter aujourd'hui, à ce sujet, les propositions de la sixième Section.

Jusqu'ici, à notre connaissance, et l'on peut s'en convaincre par l'examen des publications techniques périodiques, aide-mémoire, etc., il n'a pas été préconisé de méthode pour le choix et l'application des signes conventionnels relatifs aux installations électriques.

La représentation des schémas, au contraire, n'a eu comme règles que le goût ou l'habitude propre à chaque ingénieur ou maison de construction; la pratique a cependant rendu classiques quelques signes. La sixième Section a eu grand soin de les incorporer dans les tableaux définitifs dont elle préconise l'emploi.

C'est pour remédier à l'état de choses que nous venons d'indiquer que la sixième Section a cru bon de poursuivre l'établissement d'un systìme de signes conventionnels; disons de suite qu'elle s'est limitée aux appareils et intallations de transport, de distribution et d'utilisation d'énergie électrique, laissant de côté, au moins pour le moment, les installations de télégraphie et de téléphonie.

Il a semblé également à la sixième Section que l'uniformisation des signes conventionnels était appelée à rendre des services incontestables.

En effet, les schémas, vu le développement des installations, deviennent de jour en jour plus compliqués, et la diversité des signes choisis par les constructeurs et les exploi. tants ne peut que rendre la lecture et la compréhension plus pénibles.

Une écriture symbolique, simple, claire, facile à retenir, comportant un minimum de signes combinables entre eux, devait être relativement facile à établir pour répondreà tous les besoins; les règles à suivre devaient être également simples et d'une application très élastique. Tels sont les principes qui ont guidé la sixième Section.

Veuillez bien retenir que l'idée d'une réglementation de ce genre n'est pas nouveile.

(1) Communication de E.-J. Brunswik à la Société Internationale des Electriciens, séance du 6 février 1907. 
Elle existe en d'autres matières, notamment dans la carto. graphie où elle rend des services inestimables; de même les automobilistes ont fait œuvre analogue en multipliant sur les routes des signes conventionnels constituant de précieuses indications pour les touristes et les conducteurs; on en dirait autant de l'utilité des signaux de chemins de fer, etc.

Cependant la tâche, dans notre cas, est loin d'ètre aussi délicate, et ce qui a réussi pour ainsi dire universellement avec les uns ne peut rencontrer de sérieuses difficultés avec les autres, le but étant de même nature.

Sans prétendre à universaliser un système de signes conventionnels, la sixième Section estime qu'il n'est pas audessous du rôle de notre Société de patronner l'emploi d'un code de signes judicieusement choisis.

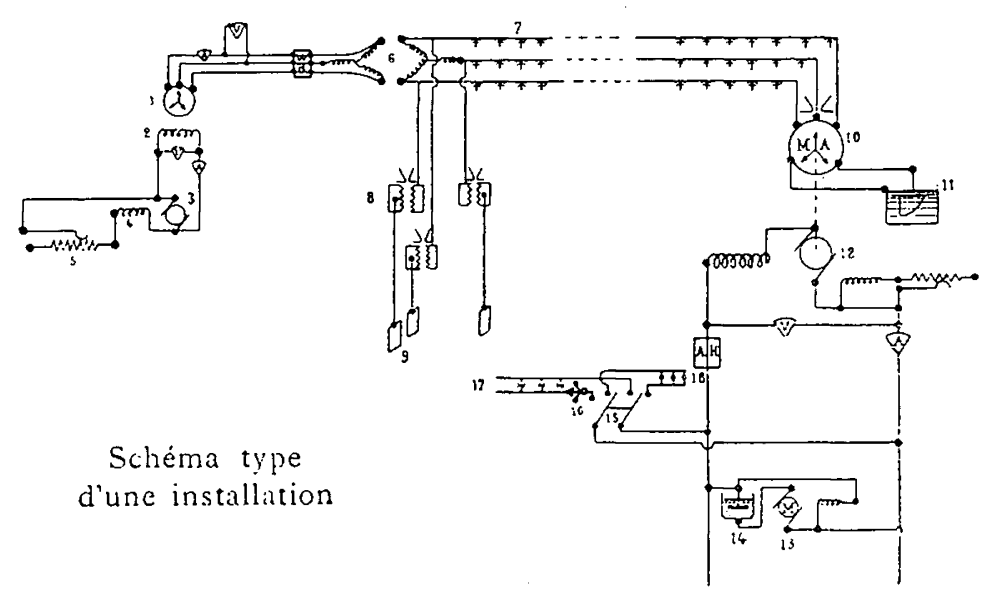

工EGEIDE

I. Alternateur triphasé.

2. Inducteurs de l'alternateur triphasé.

3. Excitatrice.

4. Irducteurs de l'excitatrice.

5. Rhéostat variable du champ de l'excitatrice.

6 . Transformateur (élévateur).

7. Ligne haute tension sur isolateurs.

8. Parafoudres haute iension.

9. Plaques de terre.

10. Moteur asynchrone triphasé; haute tension.

11. Rhéostat de démarrage (résistance variable liquide).

I2. Génératrice compound à courant continu.

I3. Moteur.

14. Résistance liquide.

15. Commutateur bipolaire à deux directions.

16. Indicateur du sens de courant.

17. Circuit d'arcs.

IS. Circuit d'incandescence.

Si nous regardons à l'étranger, nous voyons d'ailleurs que nous ne ferions en cela qu'imiter nombre de Sociétés similaires : aux Etat-Unis, les Sociétés d'assurances interviennent de très près dans le contrôle des installations électriques, et certaines obligent à établir les schémas des cana. lisations et l'appareillage avec des signes déterminés.

En Allemagne, l'Association des Electriciens allemands (V.D.E.) a publié une série de prescriptions normales concernant la figuration des schémas.

La question est donc, en quelque sorte, à l'ordre du jour. Attendre encore pour lui accorder quelque attention nous entraînerait à adopter probablement à une certaine époque un système existant, plus ou moins en accord avec nos usages et avec les besoins de méthode et de clarté chers à notre esprit.

En cette voie, la sixième Section a été d'avis : $1^{\circ}$ que les indications demandées aux signes conventionnels devaient être d'ordre tout à fait général plutôt que particulières aux détails de construction (le principe ou le but doivent être exprimés plutôt que le mode de réalisation des appareils, lequel peut varier à l'infini);

$2^{\circ}$ Que la liste des signes conventionnels ne pouvait comprendre la totalité des appareils répondant à toutes les applications susceptibles d'être traitées. La rédaction d'un Recueil de ce genre serait laborieuse, et ne serait qu'une récapitulation de catalogues; l'usage en serait à peu près impraticable;

$3^{\circ}$ Que le système à adopter devait offrir toutes ressources pour représenter, avec une suffisante clarté, un appareil quelconque par la combinaison d'un petit nombre de signes.

Par ces considérations, la sixième Section s'est arrêtée aux résolutions suivantes:

I. Le tableau des signes conventionnels dont l'emploi est à recommander ne doit comprendre :

Que le minimum possible de signes:

Que des signes faciles à reproduire à main courante comme à main posée;

Que des signes relatifs à des principes de construction.

II. Les signes conventionnels se répartissent en trois catégories :

$I^{\circ}$ Les signes fondamentaux se rapportant à un caractère commun à plusieurs classes d'appareils (voir le tableau I) ;

$2^{\circ}$ Les signes caractéristiques d'appareils usuels (poir le tableau II);

3o Les signes pouvant résulter de la combinaison des catégories précédentes pour représenter des appareils plus ou moins complexes.

TABIEAU I

SYMBOLES ET SIGNES COVENTIONNELS FONDAMENTAUX

d.ordre

Objet

Signes

conventionnels

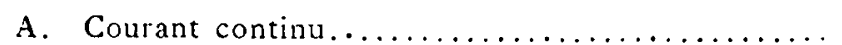

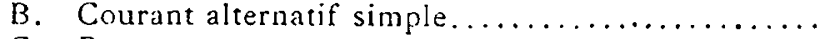

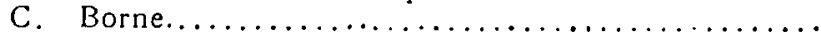

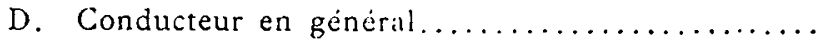

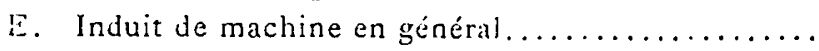

F. Dispositif quelconque de commande à distance....

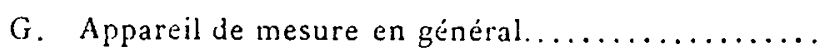

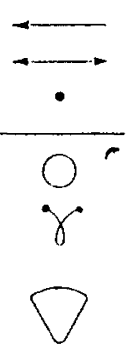

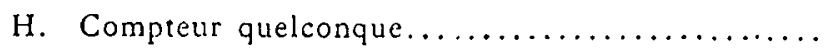

K. Dispositif à haute tension quel que soit le genre et la nature de l'appareil....................

L. Dispositif immergé dans l'huile, représentation du bain (quel que soit le genre de l'appareil)......

M. Indication dun dispositif de polarisation magnétique (")............................

()) Application à la representation de divers appareils de mesure :

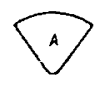

$\mathbf{I}$

I. Ampèremètre.

2. Amperemètre enregistreur. 3. Voltmètre.

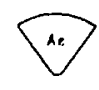

2

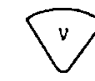

3

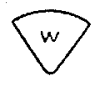

4

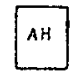

5

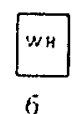

4. Wattmetre.

5. Ampere-heure-mètre 6 . Watt-heure-mètre. 
TABIEAU II

SIGIES CONYENTIONNELS GÉNÉRAUX

d'ordre

Objet

I. Interrupteur

.

2. Inverseur.

3. Disjoncteur.

4.

Coupe-circui

5. Parafoudre

6. Plaque de terre en général.

7. Commutateur

8. Réducteur daccumulateur

9. Résistance fixe en général.

10. Résistance variable solide

11. Résistance liquide en général..

12. Résistance variable liquide.

13. Bobine de self, ou solénoïde, ou inducteur........

14. Solénoïje á noyau de fer (noyau plongeur, ou circuit magnétique non fermé par du fer)..........

15. Electro-aimant.

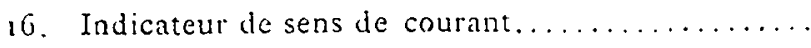

17. Induit isolé de dynamo courant continu.........

I๖. Dynamo seric.

Dynamo shunt

20.

Dỵamo compound.

21. Induit isolé, moteur à courant continu

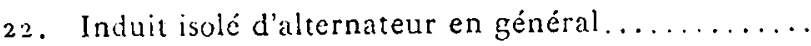

23. Alternateur synchrone:

a. Courant alternatif simple..
b. $\quad-$
diphasé
c. -
triphasé en triangle......
d. - $\quad-\quad$ triphasé en étoile (3 $\quad$ ( u 4 bornes suivant le cas)..................

24. Moteur synchrone:

a. Courant alternatif simple
b. -
diphasé.
c. -
triphasé..

25. Moteur asynchrone (bornes en nombre voulu)....

26. Transformateur (non rotatif) : (*)

Courant alternatif simple....

$27 a$. Courant alternatif triphasé (triangle)

$27 b$. Courant alternatif triphasé (étoile)

28. Transformateur diphasé.

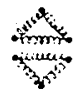

$\square$

29. Batterie daccumulateurs

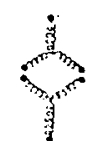

$\cdots$

3o. Batterie de piles

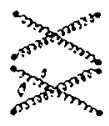

$\cdots$

3r. Lampes à arc

||$|i| i \mid i$

- - num

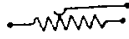

ty

古

-roon-.

事

32. Lampes à incandescence

$1.1 \cdot \% \cdot \%$

33. Isolateur basse tension.

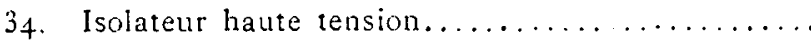

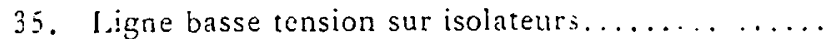

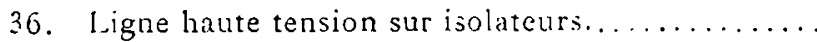

37 . Croisement de lignes.

38. Connexions

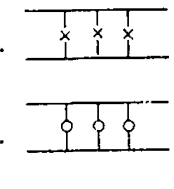

$\downarrow$<smiles>C1CCCCC1</smiles><smiles>C[C@H]1CCC2CCCC21</smiles>

Orome.<smiles>C1=CC=[GeH]C=1</smiles>

39. Poussoir, ou clé de contast.

40. Capacité

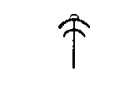

III. Les signes choisis par lạ sixieme Section sont indiqués sur les tableaux ci-joints.

Dans cette étude, la sixième Section a estimé que l'emploi des signes conventionnels devait avoir pour but de permettre la compréhension générale des schémas sans entrâ̂ner pour cela à une grande complication des dessins; il ne saurait donc, à moins de recourir à un langage hiéroglyphique plus ou moins développé, dispenser de joindre aux des sins soit des légendes, soit des instructions sur le service particulier des appareils et installations.

Se renfermant dans ce programme, la sixieme Section a pu restreindre le nombre des signes nécessaires à une solution pratique satisfaisante.

Les signes fondamentaux sont au nombre de I I ; la plupart sont déjà classiques; l'emploi facultatif de sept lettres complète cette première catégorie.

Les signes concernant les appareils les plus usuels et qu'il a paru utile de cataloguer sont au nombre de 40 seulement; ils couvrent, croyons-nous, suffisamment le champ des applications actuelles.

Ajoutons que les observations concernant l'extension des tableaux proposés seront toujours bien venues. Toutes les

(c) Le sens particulier donné, en cilectricité, au mot statique a engagé la sixième section à adopter la désignation ci-dessus pour caractériser un transformateur dont les circuits induits sont fixes, et qui ne comporte aucun organe rotatif, de préférence à l'appellation de transformateur sta ique, dont on a fait usage le plus génèralement jusqu'ici. 
communications tendant à enregistrer de nouveaux signes d'un usage courant et qui auraient pu échapper à notre nomenclature seront examinées avec la plus grande attention et donneront lieu, le cas échéant, à la publication de tableaux complémentaires. Cette méthode de révision facultative est d'ailleurs pratiquée par les Associations techniques qui élaborent des règlements.

En résumé, la sixième Section propose :

$I^{\circ}$ De préconiser et recommander l'emploi du système de signes conventionnels qui vient de vous être présenté;

$2^{\circ}$ De favoriser la propagation de ce système en lui accordant le patronage de la Société internationale des Electriciens;

$3^{\circ}$ A ce titre, d'en donner communication aux publications techniques s'occupant d'électricité industrielle ainsi qu'aux principales maisons de construction.

Ces vœux seront transmis au Bureau et au Comité de la Société internationale des Electriciens pour recevoir la suite qu'ils comporteront.

En vous les présentant, la sixième Section a l'espoir que vos efforts voudront bien se rejoindre aux siens, en vue de tirer de ce modeste travail un effet utile, et d'arriver ainsi à introduire quelque méthode et un peu de clarté dans des questions qui, quoique de mince détail $\in \mathrm{n}$ apparence, ont tout de même une certaine valeur pratique.

\section{LE MOIS HYDRO-ÉLECTRIQUE}

\section{ACADÉMIE DES SCIENCES}

\section{MÉGANIQUE ET ELEGTRIGITÉ}

Recherches expérimentales sur les diélectriques solides. Note de M. Louis Malclìs, séance du i I février 1907.

Le phénomène de la charge et de la décharge lentes des condensateurs était considéré jusqu'ici comme se manifestant à des legrés divers pour tous les diélectriques solides. On verra, par ce qui suit, que la paraffine anglaise du commerce, fusible vers $72^{\circ}$, présente une stabilité diélectrıque parfaite, c'est-à-dire n'indique ni surcharge ni résidu, et que cette propriété peut être très utilement appliquée à l'étude des diélectriques ordinaires.

Le disque de parafine est interposé entre les plateaux $\mathrm{AB}, a b$ d'un condensateur plan. L'armature $a b$, munie d'un anneau de garde $p q$, est reliée à l'une des paires de quadrants d'un électromètre Curie. L'armature AB est chargée au potentiel $+V$ d'une batterie d'accumulateurs fermée sur une forte résistance dont le milieu est au sol. L'effet sur l'électomètre est compensé par un condensateur cylindrique à capacité variable $C_{c}^{\prime}$ dont l'une des armatures $C$, chargée à - $V$, peut se déplacer de quantités connues dans la direction do l'axe. Un double contact permet de charger ou de décharger, au même instant, les deux condensateurs.

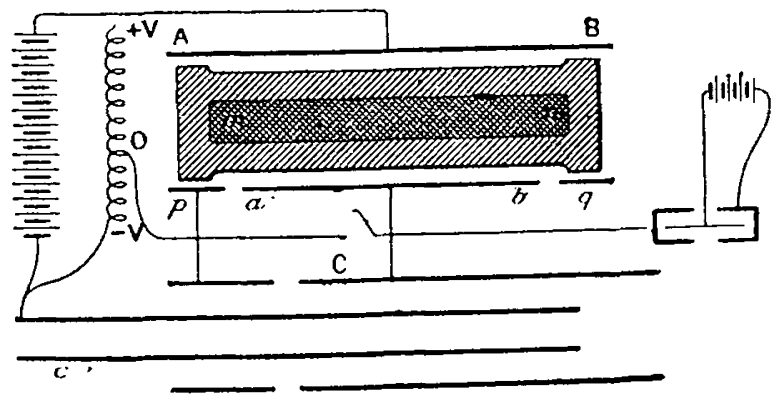

Fig. I.

Si les deux capacités ne subissent, au cours du temps, aucune variation, on peut régler leur équilibre de manière que la charge ou la décharge instantanée des deux condensateurs en opposition ne produise aucun effet sur l'électromètre. Nous avons effectué ce réglage avec l'air, puis avec la paraffine. Cette substance était d'abord interposée entre les armures du condensateur de manière à adhérer parfaitement aux surfaces $\mathrm{AB} a b$. Ensuite, nous l'avons légèrement creusée sur les deux faces, afin de supprimer le contact avec les plateaux, tout au moins Jans la région du champ sensible à l'électromètre. Nous avons opéré jusqu'à 6000 volts, l'épaisseur du diélec. trıque étant de $20 \mathrm{~mm}$. Dans tous les cas, l'équilıbre a pu être réalisé d'une manière rigoureuse, et maintenu ensuite indéfiniment. On peut en conclure que la paraffine se comporte, dans les conditions indiquées, comme un diélectrique partait.

$\mathrm{Ce}$ fait nous a suggére l'idee d'êtudier les autres diélectriques au sein de la paraffine. Nous avons éliminé ainsi les causes pertubatrices très complexes que l'on rencontre lorsqu'une couche d'air sépare un diélectrique quelconque des plateaux du condeasateur. Nos observations ont porté sur l'ébonite, le verre, le mica. Sur la figure $\mathrm{s}$, on voit le diélectrique $m n$ noyé dans de la paraffine et soumis à l'action du champ.

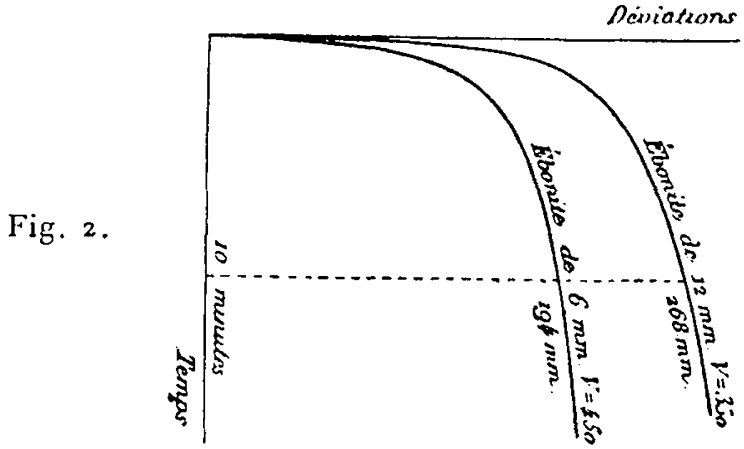

Soit à mesurer l'effet de la charge lente. Les armatures $A B$ $a b$ étant d'abord au potentiel o, on porte $\mathrm{AB}$ au potentiel $+V$, puis l'on isole, aussitòt après, le secteur de l'electometre relie à $a b$. S'il y a charge lente, l'image se déplace. On note les déviations de minute en minute. Après un temps $t$ une heure au maximuni, on ramène au 0 , d'abord $a b$, puis $A B$, et l'on isole à nouveau l'électometre. Les déviations observées donnent, dans ce cas, les valeurs de la decharge lente. Quand l'image sort de l'échelle, on a recuurs à la capacite variable pour compenser et mesurer de minute en minute les effets de la surcharge et du résidu.

Les courbes ci-dessus (fig. 2) donnent l'allure du phénomène pour deux disques d'ébonite de $12 \mathrm{~mm}$. et de $5 \mathrm{~mm}$. d'épaisseur. Ces courbes construites en portant en abscisses les minutes, et en ordonnées les déviations de l'électometre, se rapportent aussi bien à la charge qu'à la décharge lentes. La lecture $268 \mathrm{~mm}$ (fig. 2), faite aprés Io minutes de surcharge avec l'ébonite de $12 \mathrm{~mm}$, et pour $V=350$, indique un accroissement de capacité égal à $\frac{1}{15}$ de la capacité totale du condensateur plan. D'après les courbes, on voit que les déviations, extrêmement rapides au début, s'amortissent très vite. Après un temps très long, ces déviations, quoique très faibles, sont encore appréciables. Enfin, les valeurs de la charge et de la décharge lentes, au temps $t$, sont proportionnelles aux tensions.

Ainsi, avec un diélectrique solide, tel que le verre, l'ébonite, le mica, n'adhèrant pas aux armures d'un condensateur' et à l'abri, par conséquent, des effets de pénétration et de conductibilité, on observe encore une surcharge et un résidu. Nous pensons que les variatiuns de la capacité, observées dans ce cas, peuvent s'expliquer par l'existence de charges des deux signes à l'intérieur du diclectrique, et par une mobilité particulière de ces charges sous l'action du champ.

\section{GÉOLOGIE ET HYDROLOGIE}

Sur les clues de Provence, et sur les irrégularités des courbes d'équilibre des cours d'eau. - Note de M. Martel. Séance du 4 mars 1907.

En matière d'érosion par l'eau courante, nul ne conteste plus que l'intensité de l'affouillement dépende à la fois, et solidairement, de la vitesse d'écoulement et de la nature des roches attaquées. On reconnait que l'inégalité de résistance des divers terrains d'un thalweg peut faire obstacle à l'établissement régulier d'un profil d'équilibre continu, et il est même admis que les schistes tendres, par exemple, sont affouillés plus rapidement que les roches stratifiées ordinaires des calcaires.

L'étude des rivières torrentielles, effectuée, non pas par à neu près, depuis les rives ou berges, mais avec précision dans leur lit même, au milieu de l'eau et des manifestations variées qui entravent son travail, permet de bien confirmer la premiere des trois propositions ci-dessus, de généraliser considérablement la seconde, et de tenir la troisiene pour complètement erronée.

C'est, du moins, ce qui résulte de mes recherches de 1905 et 1906 au fond de plusieurs clues, ou canons des Alpes Maritimes, du Var et des Basses-Alpes. 